\title{
Candidate plant gene homologues in grapevine involved in Agrobacterium transformation
}

Research Article \author{
György D. Bisztray¹,*, Ernő Szegedi4,\# \\ ${ }^{1}$ Corvinus University of Budapest, Institute for Viticulture and Enology, \\ Department of Viticulture, 1118 Budapest, Hungary \\ ${ }^{2}$ Corvinus University of Budapest, Faculty of Horticultural Sciences, \\ Department of Genetics and Plant Breeding, 1118 Budapest, Hungary \\ 3University of Szeged, Department of Dermatology and Allergology, \\ 6720 Szeged, Hungary \\ ${ }^{4}$ Corvinus University of Budapest, Institute for Viticulture and Enology, \\ Experimental Station of Kecskemét, 6000 Kecskemét-Katonatelep, Hungary
}

Tamás Deák ${ }^{1}$, Tünde Kupi ${ }^{1}$, Róbert Oláh², Lóránt Lakatos ${ }^{3}$, Lajos Kemény ${ }^{3}$,

Received 29 January 2013; Accepted 23 April 2013

Abstract: The grapevine (Vitis vinifera) genome was analyzed in silico for homologues of plant genes involved in Agrobacterium transformation in Arabidopsis thaliana and Nicotiana spp. Grapevine homologues of the glucomannan 4-beta-mannosyltransferase 9 gene CsIA-09 involved in bacterial attachment to the cell wall, homologues of reticulon-like proteins BTI1, 2, 3 and RAB8 GTPases, both involved in T-DNA transfer to the host cell, homologues of VirE2 interacting protein VIP1 that contributes to the targeting of T-DNA into the nucleus and to its integration, and homologues of the histone protein H2A, which promotes the expression of T-DNA encoded genes, were selected. Sequences homologous to the arabinogalactan-protein AtAGP17 were not found in the grape genome. Seventeen selected candidates were tested by semiquantitative RT-PCR analysis for changes in their expression levels upon inoculation with Agrobacterium tumefaciens C58. Of the tested homologues, the expression of VvRab8a, VvVip1a and two histone genes (VvHta2 and VvHta10) increased significantly, therefore we suppose that these might be involved in Agrobacterium transformation of $V$. vinifera.

Keywords: Vitis vinifera $•$ Crown gall disease $•$ Pathogen-induced gene expression • Semiquantitative PCR

(c) Versita Sp. z 0.0

\section{Introduction}

Crown gall caused by the pathogenic Agrobacterium species is a serious disease affecting several crop plants including fruit trees, berries, ornamental plants and grapevines. However, genetic transformation of crop plants by non-tumorigenic ("disarmed") Agrobacterium tumefaciens strains has became a widely used method to introduce foreign genes into plants to improve agronomical traits [1,2].

Tumorigenic agrobacteria harbour a large plasmid called tumor inducing plasmid (pTi) that carries the genes which are essential for crown gall tumor induction. During the transformation process a region of this plasmid, the transferred DNA (T-DNA) is transported into the host cell and it becomes stably integrated into the chromosomal DNA. The T-DNA transport through a type IV secretion system [3] from the prokaryotic bacterium into the eukaryotic plant cell is determined by the virulence (vir) genes located also on the pTi, but outside the T-DNA. The T-DNA is transported in a singlestranded form (T-strand), and this process is directed by the VirD2 protein that covalently binds to the 5' end of the T-strand [2]. Additionally, for T-DNA import the VirE2 protein forms an anion selective channel on the plasma membrane of the plant cells [4] and binds to the VirD2/TDNA during the transport process. The VirD2/VirE2/Tstrand called T-complex is targeted to the nucleus of 
the plant cell. Other bacterial virulence proteins (e. g., VirE3, VirF) are also transported into the plant cells and they are involved in the integration of T-DNA [5,6].

While the introduction and nuclear targeting of T-DNA is mediated by bacterial virulence proteins, several plant proteins contribute to its integration into the plant chromosome [5,7-13]. Methods applied to identify plant genes (proteins) involved in crown gall tumorigenesis and Agrobacterium-mediated plant transformation include T-DNA tagged mutagenesis of Arabidopsis thaliana [14], virus-induced gene silencing in tobacco [7], yeast two-hybrid system $[15,16]$ and differential gene expression studies on A. thaliana, tobacco or Ageratum conyzoides plants [17-20]. These studies identified approximately 40 plant genes (proteins) which are involved in Agrobacteriummediated transformation including, for example, the Arabinogalactan-Protein AtAGP17, the Reticulon domain proteins (BTI1-3), the VirE2 interacting proteins (Vip1 and Vip2), importins, histones and several other factors involved in the ubiquitin-proteasome complex [reviewed in 10,11]. Inactivation of such genes by insertion mutagenesis or gene silencing resulted in an attenuated tumor phenotype or even resistance to Agrobacterium transformation [7,8,14-16,21-23], while their overexpression in transgenic plants increased their Agrobacterium sensitivity $[15,24,25]$. Although certain contributing genes, e. g. VIP1 and CSLA9, seem to be expressed constitutively [16,21], Agrobacteriuminfection induced the expression of a complete set of host genes involved in the transformation process [20]. For example, the histone $\mathrm{H} 2 \mathrm{~A}-1$ gene [26], the F-box protein genes VBF [23], SKP1 and SGT-1 [8] showed elevated levels of expression following inoculation with virulent or transformation competent agrobacteria. Manipulation of these contributing genes may extend the host range of Agrobacterium or result in resistance to crown gall disease $[1,11]$.

T-DNA transfer, integration and expression takes place within a relatively short period of time. For example, in tobacco cells, gusA transcripts could be detected $24 \mathrm{hrs}$ after cocultivation [27]. In Kalanchoe daigremontiana, lysopine dehydrogenase $(\mathrm{LpDH}, \mathrm{a}$ T-DNA encoded enzyme responsible for octopine synthesis) activity was detected 36 hrs after infection [28].

Genes involved in Agrobacterium transformation in grapevine (Vitis vinifera) have not been investigated yet. The proteomic analysis of grapevine embryogenic calli following cocultivation with agrobacteria showed that 69 of the identified 1100 proteins expressed differentially [29]. Their potential role in Agrobacterium-transformation was not discussed, although due to the relatively long
(3 days) cocultivation period, the contributing genes might have not been expressed anymore. Expressed sequence tag analysis in Agrobacterium-inoculated and salicylic acid-treated 'Tanmara' grapevine focused on defence related genes that may contribute to disease resistance [30].

The objective of this study was to identify the grapevine gene homologues potentially contributing to the agrobacterial transformation and to monitor the changes in their expression following cocultivation of grapevine leaf tissues with Agrobacterium cells. Based on the functional evidences from the literature, six genes were considered for detailed analysis. Arabidopsis Lysine-Rich Arabinogalactan-Protein AtAGP17 [22] and an Arabidopsis glucomannan 4-betamannosyltransferase 9 gene AtCsIA-09 [21] are both involved in the bacterial attachment to the plant cell wall. After attaching to the plant cell, the next important step of the infection is the transfer of the T-DNA into the host cell. Reticulon domain proteins BTI1 (AtRTNLB1), BTI2 (AtRTNLB2), BTI3 (AtRTNLB4), further RAB8 GTPases (AtRab8) are involved in this step from the plant side [15]. Both gene families have a high number of members in A. thaliana, 21 and 57 for AtRtnls [31] and AtGTPases [32], respectively. VirE2 interacting proteins (VIP1 and VIP2) play an important role in the infection process [33], especially VIP1, which promotes the targeting and entering of the T-DNA complex to the nucleus. VIP1 is also involved in the integration of the T-DNA into the host genome. The last step of the pathogenesis process is the expression of the transgenes. It has been shown that the H2A histone AtRat5 is involved in this step [24].

\section{Experimental Procedures}

\subsection{Selection of candidate genes for screening, primer design}

Candidate genes involved in different phases of the transformation process, such as bacterial attachment, T-DNA insertion, targeting and entering the nucleus and integration of the T-DNA into the host genome were selected from the literature $[10,15,21]$. Housekeeping genes (e. g., actin, histone $\mathrm{H} 3$, see ref. 7) which are essential for normal plant growth and development were not involved in our further studies. Amino acid sequences of the selected $A$. thaliana genes were received from the NCBI RefSeq database and homology searches were carried out using tblastn [34] against the blast database of the $12 X$ version of structural annotated coding sequences (CDS) of the homozygous 'Pinot noir' genome [35]. Multiple alignment and basic phylogenetic analysis of the $A$. thaliana reference sequence and the 
amino acid sequences of the best Blast results were carried out for each candidate using ClustalW [36]. Based on the topology of the phylogenetic tree, 2 to 5 gene family members were selected for each candidate for expression analysis.

Primers specific to the members of these gene families, that code the most similar grape peptides were designed using primer3 [37]. To ensure highest possible specificity of the primer pairs, mispriming libraries consisting of all further gene family members were defined during primer design. Primer design was verified in standard PCR reactions using DNA of the interspecific rootstock cultivar 'Richter 110' as template, except for primers incorporating intron-exon junction points or regions including large introns (data not shown). Primers used in sqPCR are listed in Table 1.

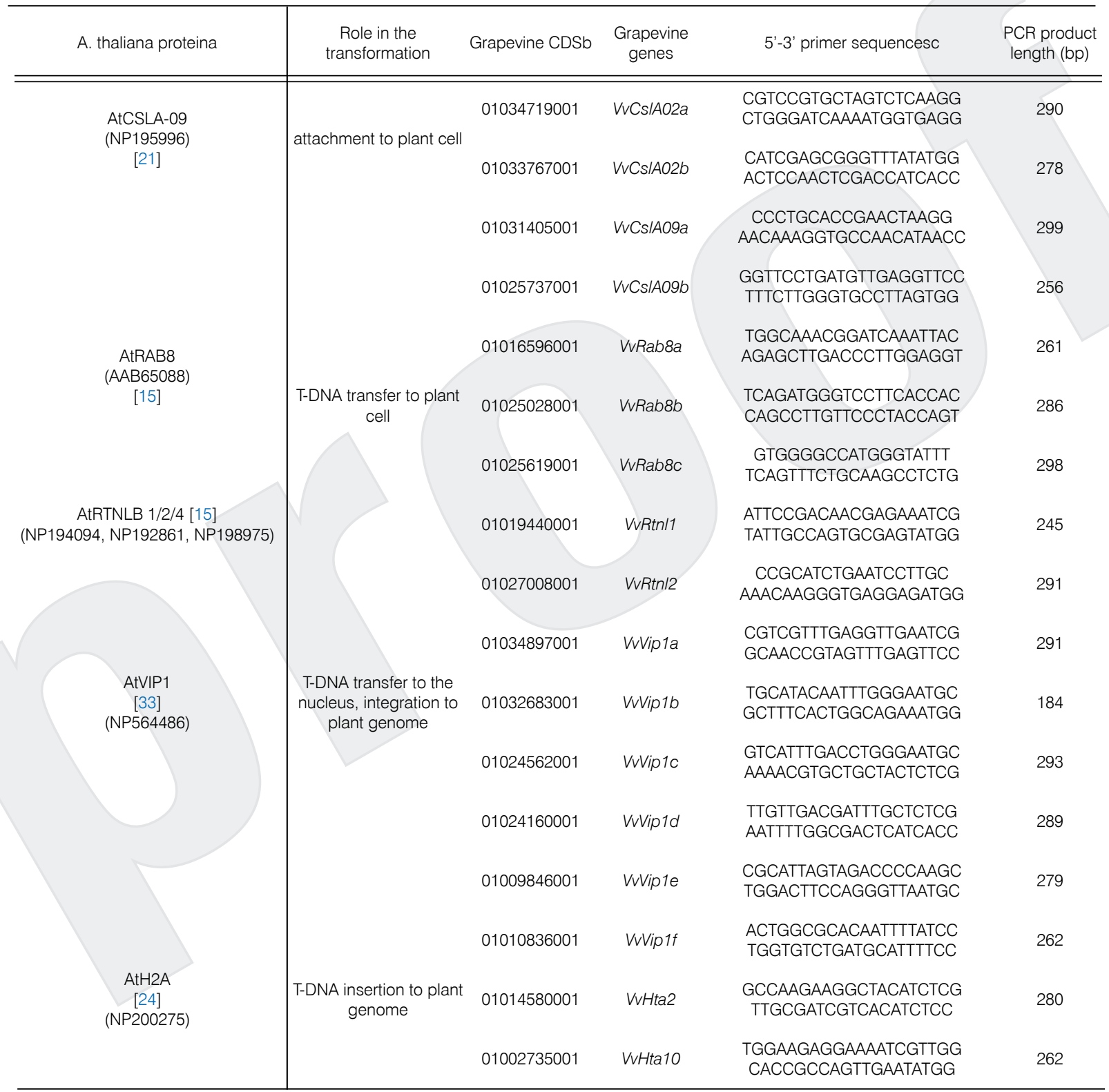

Table 1. Summary of grape coding sequences involved in the study. Names of the Arabidopsis thaliana reference genes and their roles in Agrobacterium infection are listed together with the grape homologues and the designed member-specific primers for sqPCR.

a RefSeq accession numbers and references discussing the biological function of the protein are indicated

${ }^{b}$ Identifier of the CDS in the 12X 'Pinot noir' genome annotation [35]. Eleven digit identifiers refer to annotated transcripts (GSVIVTIGrapevine CDS]) at http://www.genoscope.cns.fr/externe/GenomeBrowser/Vitis/.

c Sequence of the Forward primer is given in the upper, the Reverse primer in the lower row 


\subsection{Agrobacterium tumefaciens strain and culture conditions}

Cultures of $A$. tumefaciens $\mathrm{C} 58$ strain used for the experiments were grown overnight at $28^{\circ} \mathrm{C}$ in liquid $A B$ medium [38] supplemented with $0.5 \%(\mathrm{w} / \mathrm{v})$ glucose and $0.25 \%(\mathrm{w} / \mathrm{v})$ yeast extract. The bacterial cells were collected by centrifugation at 5,000 rpm for 10 mins, and resuspended in Tris-buffered saline (TBS) buffer (10 mM TRIS base, $150 \mathrm{mM} \mathrm{NaCl}, \mathrm{pH}=6$ ). The cell concentration was adjusted to $10^{9} \mathrm{cell} / \mathrm{ml}\left(\mathrm{OD}_{600}=1.0\right)$.

\subsection{Plant material, RNA extraction and cDNA synthesis}

Leaf discs were cut from in vitro grown $V$. vinifera cv. 'Sauvignon blanc' plants and vacuum infiltrated with $A$. tumefaciens C58 cells suspended in TBS buffer. The leaf discs infiltrated with the bacterial suspension were incubated on sterile tapwater-agar plates at $23^{\circ} \mathrm{C}$ for 36 hours (Agro), which was found appropriate in similar studies in previous works $[8,20,28]$. Control experiments were carried out using TBS buffer without bacteria under the same conditions (Mock).

RNA was extracted from the leaf discs with PureLink plant RNA reagent (Invitrogen, LifeTechnologies) according to the instructions of the manufacturer. The extracted RNA was purified from genomic DNA by using Turbo DNA-free Kit (Ambion, Life Technologies) and the final RNA concentration was adjusted to $100 \mathrm{ng} / \mu \mathrm{l}$. cDNA was synthesized using High Capacity cDNA Reverse Transcription Kit (Applied Biosystems, Life Technologies), in $20 \mu \mathrm{l}$ reaction volume from $600 \mathrm{ng}$ of total RNA, with random hexamer primers.

\subsection{Semiquantitative PCR (sqPCR) and quantitation methods}

Semi-quantitative PCR following reverse transcription (RT-sqPCR) was carried out in $20 \mu$ l reaction volume with 1x Taq buffer, $1.5 \mathrm{mM} \mathrm{MgCl}_{2}, 0.5 \mu \mathrm{M}$ of each primer, $0.2 \mathrm{mM}$ of each dNTP, $0.4 \mathrm{U}$ of Taq polymerase (Fermentas, Thermo Scientific) and $2 \mu$ of cDNA. The sqPCR cycling conditions were: $94^{\circ} \mathrm{C}$ for $2 \mathrm{~min}, 94^{\circ} \mathrm{C}$ for $30 \mathrm{~s}, 55^{\circ} \mathrm{C}$ for $30 \mathrm{~s}, 72^{\circ} \mathrm{C}$ for $30 \mathrm{~s}$, for 35 cycles. The PCR products were analyzed after agarose gel electrophoresis and ethidium bromide staining. The fluorescence intensity of Agro and Mock sample pairs binding ethidium-bromide was estimated by the ImageJ software [39]. Changes of expression levels of the investigated genes were calculated from the fluorescence intensity ratio of Agro/Mock sample pairs. The errors arising from sample preparation were corrected by normalization of target PCR product fluorescences to that of parallel $B$-actin [40] samples used as a reference gene. At least 3 biological replicates were analyzed for all candidates. Changes in the level of gene expression upon Agrobacterium infection were statistically tested using the non parametric KruskalWallis one-way analysis of variance by ranks.

\section{Results}

\subsection{Identification of genes of interest}

The first two investigated gene families are involved in attachment to the plant cell wall. Arabidopsis Lysine-Rich Arabinogalactan-Protein does not have any homologous sequences in the grape genome; as such AtAGP17 was excluded from further analysis. For glucomannan 4-beta-mannosyltransferase 9, phylogenetic analysis of 9 grape homologues of AtCsIA-09 and the A. thaliana members of the $C s / A$ and $C s / C$ families (Figure 1a) identified two grape coding sequences, which belong to the same clade as AtCsIA-09 and two further genes highly similar to AtCsIA-02 (Table 1). Four AtCsIA-09 homologues ( $V v C s I A-02 a, \quad V v C$ salA-02b, VvCsIA-09a and $V v C s / A-09 b)$ were selected for expression analysis.

The second group of plant genes contribute to the transfer of T-DNA to the plant cell. Based on the phylogenetic analysis of the $A$. thaliana family members of Reticulon domain proteins (AtRTNLB1, AtRTNLB2 and AtRTNLB4) and predicted grape coding sequences with high homology to the reference genes (data not shown), we selected 2 reticulon-like genes from grapevine for further testing (Table 1). Similarly, we compared AtRAB8 and homologous grape sequences and selected 3 Rab GTPases from grapevine for expression analysis (Table 1).

VIP1 is a bzip2 domain transcription factor involved in a broad range of the Agrobacterium infection steps. We identified 22 homologous coding sequences in the grape genome. Six of them (Table 1) were selected for expression analysis based on the phylogenetic tree drawn from AtVIP1 and the homologous grape amino acid sequences (data not shown).

Histone proteins are involved in T-DNA integration into the host genome and transgene expression. Phylogenetic analysis of 9 Arabidopsis H2A family members and 16 highly similar grapevine sequences (Figure 1b) identified two grapevine coding sequences which are possibly related to AtRAT5 (Table 1), which is known to be involved in the pathogenesis process.

\subsection{Grapevine genes showing elevated expression after Agrobacterium inoculation}

Although grapevines are mainly infected by various Agrobacterium vitis strains under natural conditions [2], we found that strains of this species cannot transform isolated plant parts (leaf discs, stem segments) in vitro. 

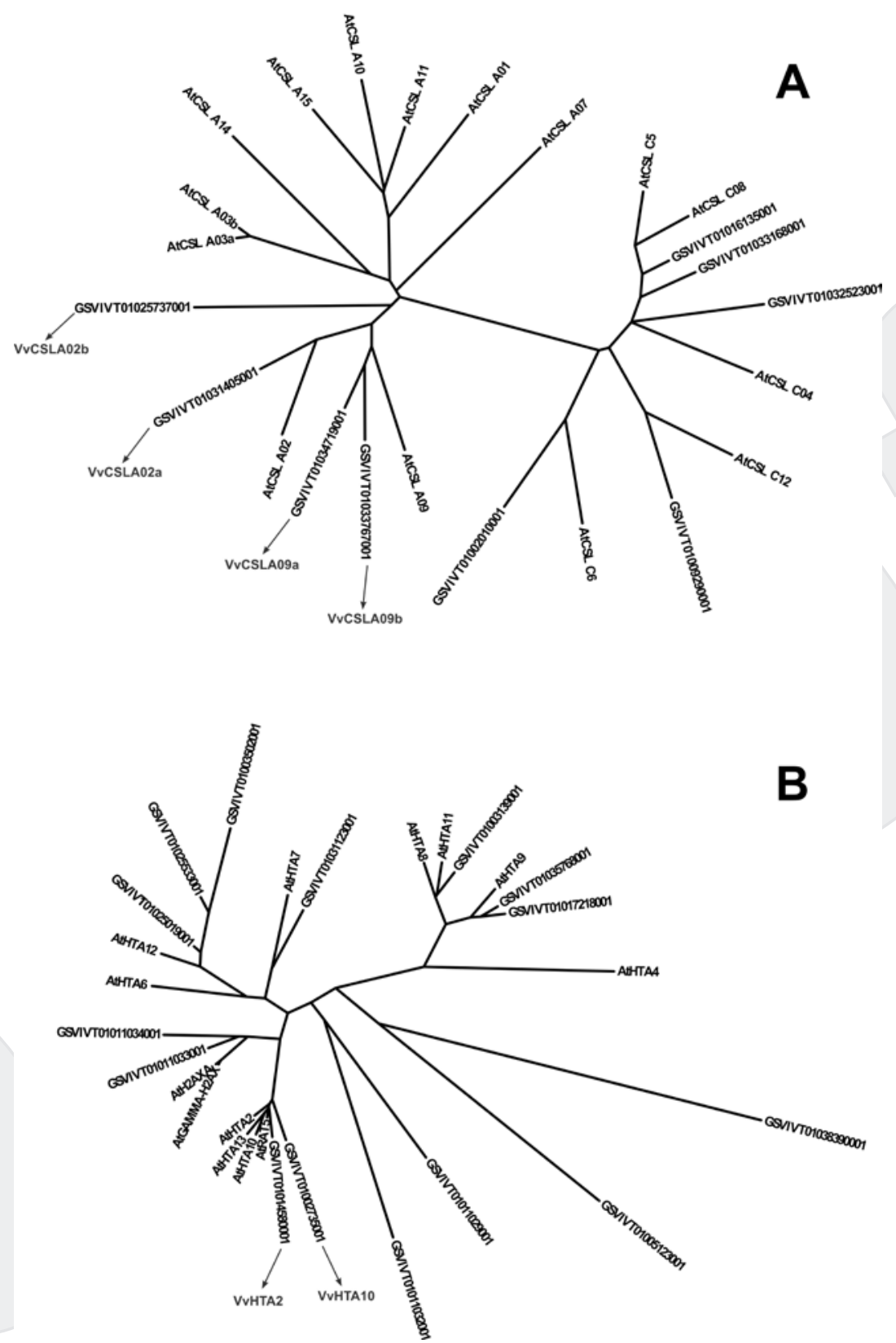

Figure 1. Unrooted neighbor-joining trees of two analyzed gene families. (A) For glucomannan 4-beta-mannosyltransferase 9 and related sequences, amino acid sequences of the CSL-A and CSL-C families from A. thaliana (AtCSL-A09 contributing to the Agrobacteriummediated genetic transformation) and homologous grape sequences were included. VvCSLA09a, VvCSLA09b, VvCSLA02a and VvCSLA02b were selected for expression analysis. (B) A. thaliana H2A histones (AtRAT5 responsible for transgene expression) and peptide products of predicted grape coding sequences similar to AtH2A were included in the analysis. VvHta2 and VvHta10 were selected for expression analysis. Grapevine CDS identifiers are indicated according to the 12X 'Pinot noir' genome annotation [35] (http://www.genoscope.cns.fr/externe/GenomeBrowser/Vitis/).

Thus we chose $A$. tumefaciens C58 strain, which was found tumorigenic on several $V$. vinifera varieties in the greenhouse and in in vitro leaf disc and stem segment transformation systems as well (our unpublished data).

During the transformation process, virulent agrobacteria up-regulate the expression of several genes that contribute to crown gall tumorigenesis
$[8,20,23,26]$. Based on these observations, we have tested 17 candidate members of five grapevine gene families by semiquantitative PCR to get data on their expression profile upon Agrobacterium infection. To this end, specific oligonucleotide sequences (Table 1) were designed for the analysis of candidate genes that may be involved in the Agrobacterium-plant interaction. 
We did not detect any expression of the chosen homologues of glucomannan 4-betamannosyltransferase 9 gene (Cs/A-02a/b; Cs/A-09a/b) either in infected or in mock inoculated grapevine leaf discs. Selected Reticulon-like and Rab8 genes - both families are involved in the T-DNA transfer into the plant cell - were expressed in infected and also in mock inoculated samples. No significant differences were found between treated and untreated samples of these genes (Figure 2a). In the case of Rab8a, the gene expression level of Agrobacterium-infected samples was significantly higher than that of mock inoculated
A
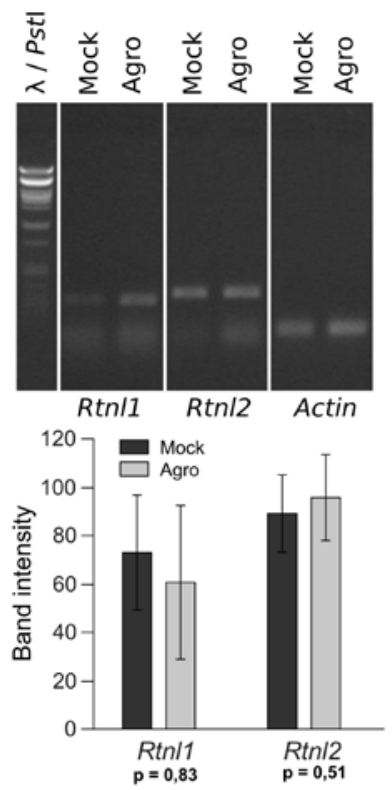

C
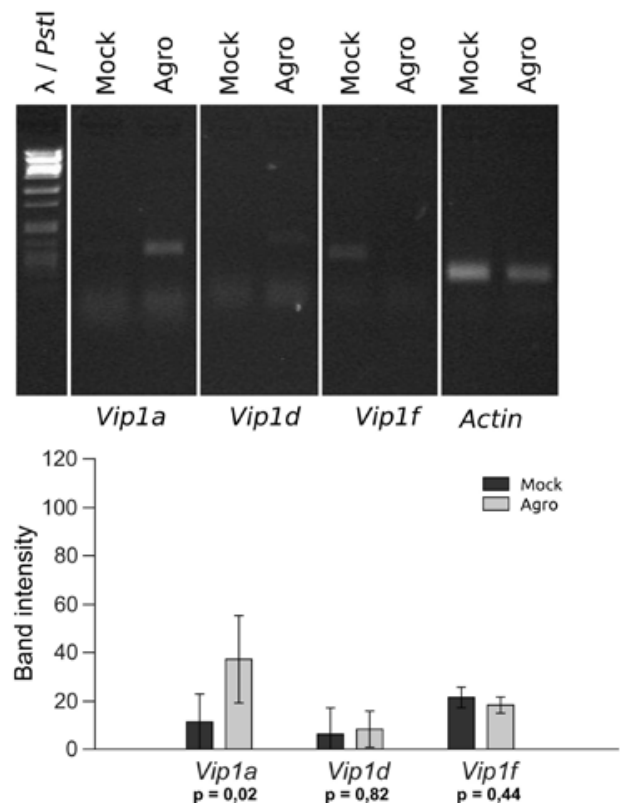

B
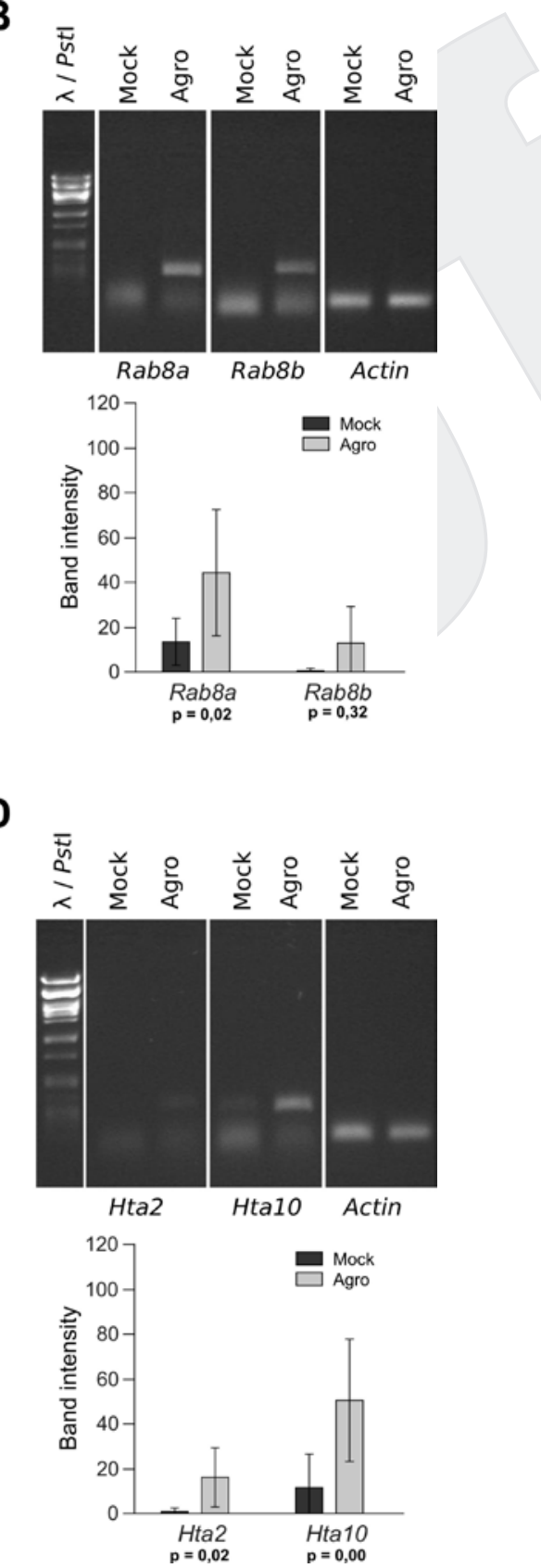

Figure 2. Changes in the expression of the candidate grapevine genes homologous to (A) Reticulon-like proteins AtRtn/1/2/4, (B) Rab8 GTPase AtRab8, (C) Vire2 Interacting protein AtVip1 and (D) H2A histone AtRat5. Expression level of each candidate was compared between mock-inoculated control samples (Mock) and leaf disks infiltrated with Agrobacterium tumefaciens (Agro). Band intensity was normalized to the expression level of the $\beta$-actin gene. Error bars represent standard deviation of biological replicates. $P$ values of the Kruskal-Wallis one-way analysis of variance by ranks between mock and Agrobacterium inoculation for the expression levels of each genes are indicated. 
samples (Figure 2b). Although Rab8b also seemed to be expressed at a higher level in treated samples, the difference was not statistically significant. Similar to $C s / A$ genes, expression of $V v R a b 8 c$ was not detected in the grapevine samples.

Similar differences, but at lower expression levels appeared in some members of the Vip1 gene family. In the case of the VvVip1a and VvVip1d genes, the treatment with Agrobacterium resulted in the increase of gene expression. For the Vip1f gene an opposite effect was observed, with a weak decrease in gene expression. However, only up-regulation of VvVip1a proved to be statistically significant (Figure 2c). Other investigated members of the gene family ( $V v V i p 1 b / c / e)$ did not show any product after sqPCR. VvHta2 and $V v H t a 10$ genes were both expressed in infected and mock inoculated samples, but Agrobacterium infection significantly increased their expression level (Figure 2d).

Altogether, 9 of the 17 investigated genes showed expression in mock or Agrobacterium inoculated leaf disk samples after 36 hours. For four genes (VvRab8a, VvVip1a, VvHta2, VvHta10) we were able to detect significant increase of the expression level after cocultivation with Agrobacterium tumefaciens.

\section{Discussion}

Crown gall disease induced by tumorigenic agrobacteria causes serious economic losses in several crop plants, including grapevines. The tumor formation is a rather complex process that includes the transfer of a well defined DNA segment, called T-DNA, from the prokaryote bacterium into the chromosome of the eukaryote host, its stable integration into the nuclear DNA and expression in the plant cell [2]. While the DNA transport and its nuclear targeting is mediated by bacterial virulence genes, several plant proteins contribute to the integration of T-DNA into the host genome $[8,10,11]$. Therefore, blocking the expression of contributing plant genes may result in resistance to Agrobacterium transformation [1,8,11,15,16], providing a potential strategy to engineer disease resistant crop plants.

An extensive in silico analysis of the grapevine genome to identify gene homologues for previously

\section{References}

[1] Gelvin S.B., Agrobacterium-mediated plant transformation: the biology behind the ,gene-jockeying” tool, Microbiol. Mol. Biol. Rev., 2003, 67, 16-37 characterized contributing genes [10] was carried out. Most of these genes are members of multigenic families, thus selecting the best candidates is rather difficult. The approach of phylogenetic analysis of the homologues offers a viable solution. However, even if candidate genes were carefully selected, there is no guarantee that the process of Agrobacterium-mediated transformation involves the same plant genes in the model organisms and in grapevine. To further narrow down the number of candidates, expression profiles of the selected genes were analyzed.

In order to collect preliminary information about changes of expression of these genes upon Agrobacterium infection, leaf discs were inoculated with A. tumefaciens C58 and the RNA fraction was analysed by semiquantitative PCR. Four ( $V v R a b 8 a$ GTPase, VvVIP1a and the histone genes VvHta2 and VvHta10) of the tested 17 homologues showed an elevated expression following cocultivation of grapevine leaf discs with Agrobacterium. For the candidates involved in the early step of the transformation process (bacterial attachment and transfer of the T-DNA), we did not find any changes in the expression levels between Agrobacterium-treated and mock-inoculated leaf discs (except of $V v R a b 8 a$ ). This may be the result of the relatively long cocultivation time. By the time of the RNA-extraction (after 36 hours cocultivation), the expression level of the genes involved in the first steps of Agrobacterium transformation were most probably already restored.

Genes showing elevated expression level after infection with Agrobacterium tumefaciens might be involved in crown gall tumorigenesis on grapevines. Further studies involving specific silencing of these genes are in progress to determine their physiological roles of in grapevine-Agrobacterium interaction to manipulate disease resistance.

\section{Acknowledgements}

This work was granted by Hungarian National Science Found (OTKA) grant no. K83121 and by TÁMOP 4 . 2. 1./B-09/01/KMR/2010-0005. We apologize to those authors whose related publications were not cited due to the space limitations.

[2] Tzfira T., Citovsky V., Agrobacterium: from biology to biotechnology, Springer Science+Business Media LLC., New York, 2008 
[3] Cascales E., Christie P.J., Definition of a bacterial type IV secretion pathway for a DNA substrate, Science, 2004, 304, 1170-1173

[4] Dumas F., Duckely M., Pelzar P., van Gelder P., Hohn B., An Agrobacterium VirE2 channel for transferred-DNA transport into plant cells, Proc. Natl. Acad. Sci. USA, 2001, 98, 485-490

[5] Citovsky V., Kozlovsky S.V., Lacroix B., Zaltsman A., Dafny-Yelin M., Vyas S., et al., Biological systems of the host cell involved in Agrobacterium infection, Cell. Microbiol., 2007, 9, 9-20

[6] Garcia-Rodríguez F.M., Schrammeijer B., Hooykaas P.J.J., The Agrobacterium VirE3 effector protein: a potential plant transcriptional activator, Nucl. Acids Res., 2006, 34, 6496-6504

[7] Anand A., Vaghchhipawala Z., Ryu C.M., Kang L., Wang K., del-Pozo O., et al., Identification and characterization of plant genes involved in Agrobacterium-mediated plant transformation by virus-induced gene silencing, Mol. Plant-Microbe Interact., 2007, 20, 41-52

[8] Anand A., Rojas C.M., Tang Y., Mysore K.S., Several components of SKP1/Cullin/F-box E3 ubiquitin ligase complex and associated factors play a role in Agrobacterium-mediated plant transformation, New Phytol., 2012, 195, 203-216

[9] Gelvin S.B., Agrobacterium in the genomic age, Plant Physiol., 2009, 150, 1665-1676

[10] Gelvin S.B., Plant proteins involved in Agrobacterium-mediated genetic transformation, Annu. Rev. Phytopathol., 2010, 48, 45-68

[11] Magori S., Citovsky V., The role of the ubiquitinproteasome system in Agrobacterium tumefaciensmediated genetic transformation of plants, Plant Physiol., 2012, 160, 65-71

[12] Pitzschke A., Hirt H., New insight into an old story: Agrobacterium-induced tumor formation in plants by plant transformation, EMBO J., 2010, 29, 10211032

[13] Tzfira T., Citovsky V., Partners in infection: host proteins involved in the transformation of plant cells by Agrobacterium, Trends Cell. Biol., 2002, 12, 121-129

[14] Zhu Y., Nam J., Humara J.M., Mysore K.S., Lee L.-Y., Cao H., et al., Identification of Arabidopsis rat mutants, Plant Physiol., 2003, 132, 494-505

[15] Hwang, H.-H., Gelvin, S. B., Plant proteins that interact with VirB2, the Agrobacterium tumefaciens pilin protein, mediate plant transformation, Plant Cell, 2004, 16, 3148-3167

[16] Tzfira T., Vaidya M., Citovsky V., VIP1, an Arabidopsis protein that interacts with Agrobacterium VirE2, is involved in VirE2 nuclear import and Agrobacterium infectivity, EMBO J., 2001, 20, 3596-3607

[17] Ditt R.F., Nester E.W., Comai L., Plant gene expression response to Agrobacterium tumefaciens, Proc. Natl. Acad. Sci. USA, 2001, 98, 10954-10959

[18] Ditt R.F., Kerr K.F., de Figueiredo P., Delrow J., Comai L., Nester E.W., The Arabidopsis thaliana transcriptome in response to Agrobacterium tumefaciens, Mol. Plant-Microbe Interact., 2006, 19, 665-681

[19] Lee C.-W., Efetova M., Engelmann J.C., Kramell R., Wasternack C., Ludwig-Müller J., et al., Agrobacterium tumefaciens promotes tumor induction by modulating pathogen defense in Arabidopsis thaliana, Plant Cell, 2009, 21, 29482962

[20] Veena, Jiang H., Doerge R.W., Gelvin S.B., Transfer of T-DNA and Vir proteins to plant cells by Agrobacterium tumefaciens induces expression of host genes involved in mediating transformation and suppresses host defense gene expression, Plant J., 2003, 35, 219-236

[21] Zhu Y., Nam J., Carpita N.C., MatthysseA.G., Gelvin S.B., Agrobacterium-mediated root transformation is inhibited by mutation of an Arabidopsis cellulose synthase-like gene, Plant Physiol., 2003, 133, 1000-1010

[22] Gaspar Y.M., Nam J., Schultz C.J., Lee L.-Y., Gilson P.R., Gelvin S.B., et al., Characterization of the Arabidopsis lysine-rich arabinogalactan-protein AtAGP17 mutant (rat1) that results in a decreased efficiency of Agrobacterium transformation, Plant Physiol., 2004, 135, 2162-2171

[23] Zaltsman A., Krichevsky A., Loyter A., Citovsky V., Agrobacterium induces expression of a host F-box protein required for tumorigenicity, Cell Host \& Microbe, 2010, 7, 197-209

[24] Tenea G.N., Spantzel J., Lee L.-Y., Zhu Y., Lin K., Johnson S.J., et al., Overexpression of several Arabidopsis histone genes increases Agrobacterium-mediated transformation and transgene expression in plants, Plant Cell, 2009, 21, 3350-3367

[25] Tzfira T., Vaidya M., Citovsky V., Increasing plant susceptibility to Agrobacterium infection by overexpression of the Arabidopsis nuclear protein VIP1, Proc. Natl. Acad. Sci. USA., 2002, 99, 1043510440

[26] Yi H.C., Mysore K.S., Gelvin S.B., Expression of the Arabidopsis histone $\mathrm{H} 2 \mathrm{~A}-1$ gene correlates with susceptibility to Agrobacterium transformation, Plant J., 2002, 32, 285-298 
[27] Narasimbulu S.B., Deng X.-B., Sarria R., Gelvin S.B., Early transcription of Agrobacterium T-DNA genes in tobacco and maize, Plant Cell, 1996, 8, 873-886

[28] Otten L., Lysopine dehydrogenase activity as an early marker in crown gall transformation, Plant Sci. Lett., 1982, 25, 15-27

[29] Zhao F., Chen L., Perl A., Chen S., Ma H., Proteomic changes in grape embryogenic callus in response to Agrobacterium tumefaciens-mediated transformation, Plant Sci., 2011, 181, 485-495

[30] Choi Y.J., Yun H.K., Park K.S., Noh J.H., Heo Y.Y., Kim S.H., et al., Transcriptional profiling of ESTs responsive to Rhizobium vitis from 'Tanmara' grapevines (Vitis sp.), J. Plant Physiol., 2010, 167, 1084-1092

[31] Nziengui H., Bouhidel K., Pillon D., Der C., Marty F., Schoefs B., Reticulon-like proteins in Arabidopsis thaliana: structural organization and ER localization, FEBS Lett., 2007, 581, 3356-3362

[32] Rutherford S., Moore I., The Arabidopsis Rab GTPase family: another enigma variation, Curr. Opin. Plant Biol., 2002, 5, 518-528

[33] Li J., Krichevsky A., Vaidya M., Tzfira T., Citovsky V., Uncoupling of the functions of the Arabidopsis VIP1 protein in transient and stable plant genetic transformation by Agrobacterium, Proc. Natl. Acad. Sci. USA., 2005, 102, 5733-5738
[34] Altschul S.F., Gish W., Miller W., Myers E.W., Lipman D.J., Basic local alignment search tool, J. Mol. Biol., 1990, 215, 403-410

[35] Jaillon O., Aury J.-M., Noel B., Policriti A., Clepet C., Casagrande A., et al., The grapevine genome sequence suggests ancestral hexaploidization in major angiosperm phyla, Nature, 2007, 449, 463-468

[36] Larkin M.A., Blackshields G., Brown N.P., Chenna R., McGettigan P.A., McWilliam H., et al., ClustalW and ClustalX version 2, Bioinformatics, 2007, 23, 2947-2948

[37] Rozen S., Skaletsky H., Primer3 on the WWW for general users and for biologist programmers, In: Krawetz S., Misener S. (Eds.) Bioinformatics Methods and Protocols: Methods in Molecular Biology, Humana Press, Totowa, 2000

[38] Lichtenstein C., Draper J., Genetic engineering of plants, In: Glover D.M. (Ed.) DNA cloning: a practical approach, Vol. II., IRL Press, Oxford, 1986

[39] Schneider C.A., Rasband W.S., Eliceiri K.W., NIH Image to ImageJ: 25 years of image analysis, Nature Methods, 2012, 9, 671-675

[40] Reid K.E., Olsson N., Schlosser J., Peng F., Lund S.T., An optimized grapevine RNA isolation procedure and statistical determination of reference genes for real-time RT-PCR during berry development, BMC Plant Biol., 2006, 6, 27 